\title{
BKlotho Inhibits Cell Proliferation by Downregulating ELK4 and Predicts Favorable Prognosis in Prostate Cancer
}

\author{
Changlin Mao' \\ Wei Dong ${ }^{2}$ \\ Jiaju $\mathrm{Lu}^{3}$ \\ Zhao Zhang ${ }^{4}$ \\ Hongliang $\mathrm{Wu}^{5}$ \\ Armin Ghavamian' \\ Dongbin $\mathrm{Bi}^{3}$ \\ Pei Gao' \\ Zhao Liu $^{5}$ \\ Sentai Ding ${ }^{1,3}$ \\ 'Department of Urology, Shandong \\ Provincial Hospital, Cheeloo College of \\ Medicine, Shandong University, Jinan, \\ Shandong, 25002I, People's Republic of \\ China; ${ }^{2}$ Department of Urology, \\ Shandong Provincial Hospital West \\ Branch, Jinan, Shandong, 250000, People's \\ Republic of China; ${ }^{3}$ Department of \\ Urology, Shandong Provincial Hospital \\ Affiliated to Shandong First Medical \\ University, Jinan, Shandong, 25002I, \\ People's Republic of China; ${ }^{4}$ Department \\ of Urology, The Affiliated Hospital of \\ Qingdao University, Qingdao, Shandong, \\ 266000, People's Republic of China; \\ ${ }^{5}$ Department of Urology, Qilu Hospital, \\ Cheeloo College of Medicine, Shandong \\ University, Jinan, Shandong, 2500I2, \\ People's Republic of China
}

Correspondence: Zhao Liu

Department of Urology, Qilu Hospital, Cheeloo College of Medicine, Shandong University, Jinan, Shandong, 2500I2, People's Republic of China

Email zhao.liu@sdu.edu.cn

Sentai Ding

Department of Urology, Shandong Provincial Hospital Affiliated to Shandong First Medical University, Jinan, Shandong, 25002I, People's Republic of China Email dingsentai@I26.com
Objective: Prostate cancer ( $\mathrm{PCa}$ ) ranks as the second common malignancy in males worldwide. Although conspicuous progressions in diagnosis and treatment have been achieved in the past decades, the prognosis expectation of PCa remains unsatisfied yet. To improve the prognosis prediction of $\mathrm{PCa}$, more specific biomarkers are needed. In this retrospective research, we focused on $\beta$ Klotho and ETS-like transcription factor 4 (ELK4), aiming to identify potential prognostic biomarkers for PCa.

Methods: Western blotting was used to determine the expression of $\beta$ Klotho, ELK4, and PARP in C4-2B and PC3 PCa cell lines. CCK-8 assay and colony formation assay were applied to examine the roles of $\beta$ Klotho and ELK4 in the proliferation of PCa cells. The expression of $\beta$ Klotho and ELK4 in PCa tissue samples was determined by immunochemistry. Pearson's $\chi 2$ test and Fisher's exact test were performed to investigate the associations among $\beta$ Klotho, ELK4 and various clinical factors. Kaplan-Meier curves and Cox regression model were established to reveal the correlation among $\beta$ Klotho, ELK4 expression and the prognosis of patients.

Results: $\beta$ Klotho overexpression down-regulated the ELK4 expression, induced apoptosis and inhibited cell proliferation in both $\mathrm{C} 4-2 \mathrm{~B}$ and PC3 cells, which were reversed by ELK4 overexpression. $\beta$ Klotho expression in $\mathrm{PCa}$ tissue samples had negative correlation with the ELK4 expression, and higher $\beta$ Klotho expression was associated with lower Gleason score, absent distant metastasis and lower prostate-specific antigen (PSA) level. On the contrast, higher ELK4 expression was correlated with distant metastasis and higher PSA level. Moreover, $\beta$ Klotho and ELK4 were both recognized as independent factors for the prognosis of patients with $\mathrm{PCa}$.

Conclusion: $\beta$ Klotho inhibits proliferation of prostate cancer cells by downregulating ELK4. Both $\beta$ Klotho and ELK4 expressions correlate with the prognosis of PCa, which may serve as potential biomarkers for follow-up surveillance and prognostic assessments.

Keywords: FGFR, biomarker, prostate cancer, survival

\section{Introduction}

Prostate cancer (PCa) is considered as the most diagnosed cancer for men in 2020 worldwide, with 191,930 new cases, accounting for almost $21.5 \%$ newly diagnosed cases in all types of cancer, the total deaths were up to 33,330, as a proportion of $10 \% .^{1}$ Over the past decades, a steady increasement of PCa incidences and mortality in Asian countries was observed, due to the changes in risk factor exposure, westernized lifestyle tendency and insufficient cancer screenings. ${ }^{2}$ Especially in 
China, the diagnosis of PCa was evermore at later stage than western countries, with the mortality rate of 42.2 per million in 2018. ${ }^{3}$

Although tremendous progressions in $\mathrm{PCa}$ managements have been achieved during the past decades, due to the highly heterogeneous $\mathrm{PCa}$, the unpredictability of disease evolutions and outcomes is still the major therapeutic challenge. Prostate-specific antigen (PSA), as the clinical widely applied biomarker, significantly improved $\mathrm{PCa}$ detection rate and decreased mortality. However, the screening of serum PSA also resulted in a controversial dilemma related to overdiagnosis and overtreatment, especially for indolent low-risk PCa. ${ }^{4}$ Although a series of markers for PCa have recently been investigated, such as osteopontin, ${ }^{5}$ RRBP $1,{ }^{6}$ and $\mathrm{FKBP} 9,{ }^{7}$ there still remains insufficient diagnostic and prognostic tools for use in clinical settings. Therefore, the identification of effective biomarkers for predicting the prognosis of $\mathrm{PCa}$ and establishing promising therapeutic approaches are required.

Endocrine fibroblast growth factor (FGF) receptor complex is formed by the high-affinity conjunction of FGF21, FGF19, and FGF23 with aklotho or BKlotho, guided by their FGFRs. Collectively, these essential components build a unique endocrine regulation system. ${ }^{8}$ $\beta K$ lotho, an essential coreceptor of the FGF/FGFR signaling pathway, is characterized as a single-pass transmembrane protein composed of 1014 amino acids. ${ }^{9}$ Growing evidence indicated that the FGF- $\beta$ Klotho-FGFR endocrine system has a crucial role in the pathophysiology of various diseases, including diabetes, arteriosclerosis and chronic kidney failure. ${ }^{10,11}$ The initiation and progression of multiple carcinomas were also reported to be linked to $\beta$ Klotho. ${ }^{12-14}$ Moreover, our previous studies revealed that the expression of $\beta$ klotho in PCa tissue was lower than that in benign prostate tissue, and $\beta$ klotho overexpression significantly inhibited the epithelial-mesenchymal transition (EMT) process of $\mathrm{PCa} .{ }^{15}$ However, the value of $\beta$ Klotho expression in predicting the clinical prognosis of PCa has not been elucidated yet.

ELK4 (SRF accessory factor-1, SAP-1) belongs to the ELK subfamily of the ETS transcription factors that are classic targets for mitogen-activated protein kinases (MAPKs)/ERK signaling. ${ }^{16}$ Recently, various studies have detailedly elucidated the crucial role of ELK4 participating in various metabolic procedures: cell differentiation, ${ }^{17}$ inflammatory response $^{18}$ and cellular microenvironment regulation. ${ }^{19}$ The oncogenic role of ELK4 was also verified in multiple cancers, ${ }^{20,21}$ including
PCa. ${ }^{16,22}$ In addition, ELK4 was identified as a crucial nuclear cofactor of ERK signaling pathway, acting adequately in coordination with their DNA targeting partner SRF. ${ }^{17}$ We previously reported that $\beta$ Klotho overexpression inactivates ERK signaling in endometrial cancer and PCa. ${ }^{13,15}$ However, whether there is a regulatory relationship between $\beta K$ lotho and ELK4, and the role of ELK4 in $\mathrm{PCa}$ prognosis has not been reported.

In this study, we investigated the expressions of $\beta K$ lotho and ELK4, and their potential regulatory pattern in $\mathrm{PCa}$ tissues and cells, respectively. Meanwhile, we evaluated the clinicopathological significance and prognostic values of $\beta$ Klotho and ELK4 in PCa.

\section{Materials and Methods}

\section{Patient Selection and Tissue Samples}

This was a retrospective study, which enrolled $109 \mathrm{PCa}$ patients from Shandong Provincial Hospital Affiliated to Shandong First Medical University from October 2012 to December 2016. All tissue samples were obtained from prostatectomy or biopsy, fixed in $4 \%$ buffered formalin and embedded in paraffin. The slides were stained with hematoxylin and eosin, analyzed by two independent board-certified pathologists and selected the most representative slide for each case. Clinicopathological data, including age, Gleason scores, distant metastasis status, lymphatic status, and PSA levels, were collected. None of the patients recruited in this study had received neoadjuvant chemotherapy, radiotherapy or hormonal therapy before. Those who were diagnosed with an extra malignant carcinoma or other life-threatening disease were excluded from this study, all the patients enrolled in this study had an Eastern Cooperative Oncology Group (ECGO) performance status score less than 3 points (will be tolerant to surgeries or adjuvant therapies). For localized $\mathrm{PCa}$, patients with low, intermediate or a proportion highly selected high-risk PCa were recommended for radical prostatectomy, adjuvant therapies (hormonal treatment or radiation treatment) were applied to remain high-risk PCa patients, also referring to their individual preferences. For metastatic $\mathrm{PCa}$, patients were performed biopsies followed by androgen deprivation therapy (ADT), with or without chemotherapy. The follow-up scheme was executed every three months by multiple approaches (telephone, e-mail, outpatient), the median follow-up time was 44.84 months (ranges: 3 to 92 months). Overall survival (OS) was defined as the time from surgical 
procedures to death or the last follow-up and cancerspecific survival (CSS) was defined as the time from surgical procedures to death caused by $\mathrm{PCa}$ or the last follow-up. This study complies with the Declaration of Helsinki. All patients signed informed consent prior to study commencement and this study was approved by the Medical Ethics Committee of Shandong Provincial Hospital Affiliated to Shandong First Medical University.

\section{Immunohistochemistry}

The protocol and analysis of immunohistochemistry were performed as previously described. ${ }^{14}$ The fresh tissues were washed with phosphate-buffered saline (PBS) and then fixed in $4 \%$ paraformaldehyde. After dehydration and paraffin embedding, the samples were cut into $5 \mu \mathrm{m}$ sections and mounted onto glass slides. The deparaffinized, rehydrated sections were incubated with $3 \% \mathrm{H} 2 \mathrm{O} 2$ and then antigen retrieval was performed. After blocking, the sections were incubated overnight with the anti$\beta$ Klotho (diluted 1:100, Abcam, cat. no. 106794), and antiELK4 (diluted 1:100, Santa Cruz, cat. no. sc-166823) primary antibodies in a humid chamber at $4^{\circ} \mathrm{C}$. HRP conjugated goat anti-rabbit and anti-mouse IgG (ZSGB-BIO, cat. no. SAP-9100) was used as the secondary antibody.

The immunohistochemical scores were evaluated according to the staining intensity and percentage of stained cells. The staining intensity was judged as follows: colorless, 0 points; light yellow, 1 point; brown, 2 points; and tan, 3 points. The percentage of stained cells was scored as follows: $<5 \%, 0$ points; $5 \% \sim 25 \%, 1$ point; $26 \% \sim 50 \%, 2$ points; $51 \% \sim 75 \%, 3$ points; and $76 \%$ $100 \%, 4$ points. The two scores were multiplied to obtain the immunohistochemical scores: $\leq 6$ was defined as low, and $>6$ was defined as high.

\section{Cell Culture and Transfection}

We obtained the human PCa cell lines C4-2B and PC3 from the American Type Culture Collection (ATCC, Manassas, USA), and the short-tandem repeat (STR) assay (Microread, Beijing, China) was performed to verify the cell line. We cultured the C4-2B and PC3 cells in RPMI-1640 medium (Invitrogen, Carlsbad, USA), supplemented with $100 \mathrm{U} / \mathrm{mL}$ penicillin, $100 \mu \mathrm{g} / \mathrm{mL}$ streptomycin and $10 \%$ fetal bovine serum (FBS), at the temperature of $37^{\circ} \mathrm{C}$ with a $5 \% \mathrm{CO}_{2}$ humidified environment. The cells were cultured on the 6-well plates and transfected with $2 \mu \mathrm{g}$ of the GV230-CON083 plasmid, GV230- $\beta$ Klotho plasmid, or equivalent GV230- $\beta$ Klotho plus GV230-ELK4 plasmid by using the X-tremeGENE HP DNA Transfection reagent (Roche, Rotkreuz, Switzerland) according to the manufacturer's protocol. All plasmids were constructed by GeneChem Co., Ltd. (Shanghai, China).

\section{Protein Extraction and Western Blotting Assay}

The cells were collected by centrifugation $\left(4{ }^{\circ} \mathrm{C}\right.$, 12,000 rpm, $5 \mathrm{~min}$ ) and then washed by PBS for twice. RIPA lysis buffer containing Protease inhibitor (Thermo, Rockford, USA) was applied for protein extraction. Thereafter, protein lysate sample was subjected to $10 \%$ SDS-PAGE for electrophoretically separation and transferred to the nitrocellulose membrane, followed by blocking with sealing solution (TBST solution containing $5 \%$ skim milk) at room temperature for $1 \mathrm{~h}$. After incubation with the primary antibodies and subsequently incubation with the peroxidase-conjugated secondary antibodies, the protein band was visualized by the ECL Western blotting system (BD Biosciences, USA). $\beta$-actin was used as the loading control in this assay. The anti$\beta$ Klotho (cat. no. ab106794) was purchased from Abcam (Cambridge, MA, USA), anti-ELK4 (cat. no. sc-166823) and anti-PARP (cat. no. sc-53643) antibodies were purchased from Santa Cruz Biotechnology (Santa Cruz, CA, USA). Anti- $\beta$-actin (cat. no. TA-09) antibody was purchased from ZSGB BIO (Beijing, China) and secondary antibodies (cat. no. SA00001-1 and SA00001-2) were purchased from WUHAN SANYING (Wuhan, China).

\section{Cell Counting Kit-8 (CCK-8) Assay}

We applied cell counting kit-8 (Tongren, Shanghai, China) to assess the cell proliferation. The cells in the logarithmic phase were digested and formulated into cell suspensions, afterward, laid on the 96-well plate with a density of 1000 cells/well, a measure of $10 \mu \mathrm{L} 5 \mathrm{mg} / \mathrm{mL} \mathrm{CCK}-8$ regent was added to the plate well at the $0 \mathrm{~h}, 24 \mathrm{~h}, 48 \mathrm{~h}$ and $72 \mathrm{~h}$ after incubation. The culture was terminated 1 hour after CCK8 regent adding, and the optical density OD value of each well was detected by microplate reader (Tecan, Mannedorf, Switzerland) at $450 \mathrm{~nm}$. The experiments were repeated in triplicate for each group.

\section{Colony Formation Assay}

The cells were inoculated on the 6-well plates with a density of 500 cells/well in triplicate in $3 \mathrm{~mL}$ of medium 
containing $10 \%$ FBS and allowed to grow for 7 days. The culture medium was replaced every 3 days. Following incubation, the medium was removed. The colonies were fixed with $4 \%$ paraformaldehyde for $15 \mathrm{~min}$, and then stained with hematoxylin for $15 \mathrm{~min}$. The stained cells were rinsed three times with tap water to remove the excess dye. Each dish was then washed and dried. The colonies with diameter larger than $1.5 \mathrm{~mm}$ were counted.

\section{Statistics}

SPSS 25.0 software (SPSS Inc., Chicago, IL, USA) was used for statistical analysis. The numerical data were presented as the means $\pm \mathrm{SD}$ and the differences between the two groups were determined by the two-tailed Student's $t$-test. The comparison of categorical variables was performed by using the chi-square test $\left(\chi^{2}\right)$ or Fisher's exact test. Kaplan-Meier curves and Cox regression model were established to reveal the correlation among $\beta$ Klotho, ELK4 expression and the prognosis of patients. A value of $p<$ 0.05 was considered statistical significance.

\section{Results}

\section{$\beta$ Klotho Overexpression Inhibited the} Proliferation and Promoted Apoptosis of Prostate Cancer Cells by Downregulating ELK4

The Western blotting assay was conducted to determine the expression of $\beta$ Klotho, ELK4 and PARP in PCa C4$2 \mathrm{~B}$ and PC3 cells. The results showed that $\beta$ Klotho overexpression significantly down-regulated the expression of ELK4, and the $89 \mathrm{kDa}$ cleavaged PARP band was appeared following $\beta$ Klotho overexpression, which indicated that the elevated $\beta$ Klotho expression induced the apoptosis of PCa cells. In addition, after introduction of ELK4, the expression of cleavaged PARP was decreased (Figures 1A and 2A). By using CCK-8 assay, we showed that $\beta$ Klotho overexpression significantly reduced the proliferation of both $\mathrm{C} 4-2 \mathrm{~B}$ and $\mathrm{PC} 3$ cells, which was reversed by the exogenous ELK4 expression (Figures $1 \mathrm{~B}$ and 2B). The effects of $\beta$ Klotho and ELK4 on cell proliferation were further demonstrated by colony formation assay (Figures 1C and $2 \mathrm{C})$.

\section{$\beta$ Klotho Expression Negatively Correlate with ELK4 Expression in PCa Tissues}

The immunohistochemical staining was conducted to determine the expression level of $\beta$ Klotho and ELK4 in PCa tissues from 109 patients. Representative high and low immunohistochemical staining of $\beta$ Klotho and ELK4 are shown in Figure 3A. The majority of $\beta$ Klotho was expressed in the cytoplasm and cytomembrane of both epithelial and stromal cells, whereas the ELK4 was mainly located in the nucleus of epithelial cells. Besides, the Pearson correlation coefficient analysis showed a significantly negative association between $\beta$ Klotho and ELK4 expression $(r=-0.425, p<0.001$, Figure 3B). Patients with low $\beta$ Klotho expression were more likely to show high ELK4 expression, and vice versa (Figure 3C). These results indicated that the expression of $\beta$ Klotho was inversely correlated with ELK4.

\section{Clinicopathologic Parameters of the Cases}

At the end of the study, 72 (66.1\%) patients were alive, $33(30.3 \%)$ patients were died, and $4(3.67 \%)$ patients were lost to follow-up. We retrospectively collected the clinicopathological information (including age, Gleason score, $\mathrm{T}$ stage, lymphatic status, distant metastasis and PSA level) of the patients, and the results are listed in Table 1. The median age of patients was 67 years (range: 52-86 years). Forty-three (39.4\%) patients were less or equal to 65 years, $66(60.6 \%)$ patients were above 65 years. Twenty-five $(22.9 \%)$ patients were identified to be with positive lymphatic status and $20(18.3 \%)$ patients were found to possess distant metastasis (organ or bone). Twenty-two (20.2\%) patients were at tumor stage T1, 50 (45.9\%) patients were at tumor stage T2, 27 (24.8\%) patients were at tumor stage T3 and $10(9.2 \%)$ patients were at tumor stage T4. Sixty-six (60.6\%) patients had a Gleason score below or equal to 7 and 43 (39.4\%) patients had a Gleason score above or equal to 8 . A total of $23(21.1 \%)$ patients had a PSA level less than $10 \mathrm{ng} / \mathrm{mL}, 36(33.0 \%)$ patients measured between 10 and $20 \mathrm{ng} / \mathrm{mL}$ and 50 (45.9\%) patients had a PSA level above $20 \mathrm{ng} / \mathrm{mL}$. 
A

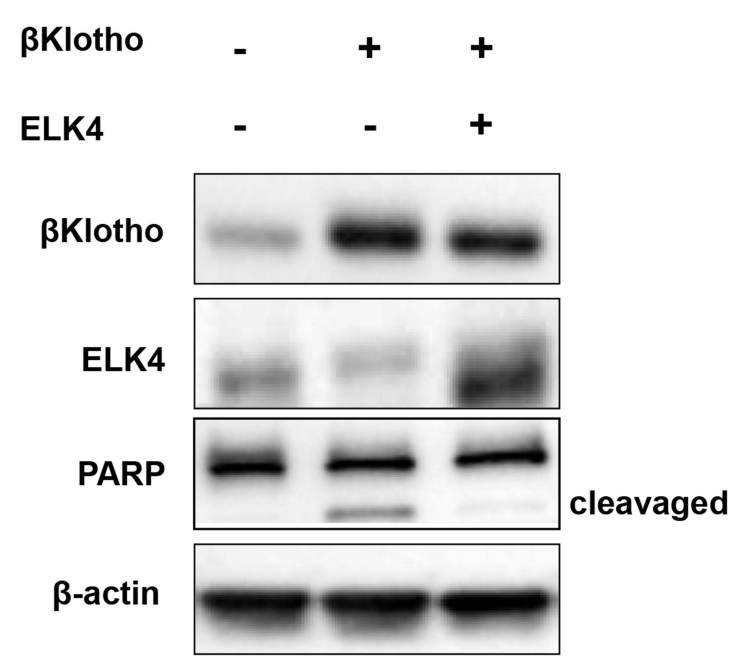

B

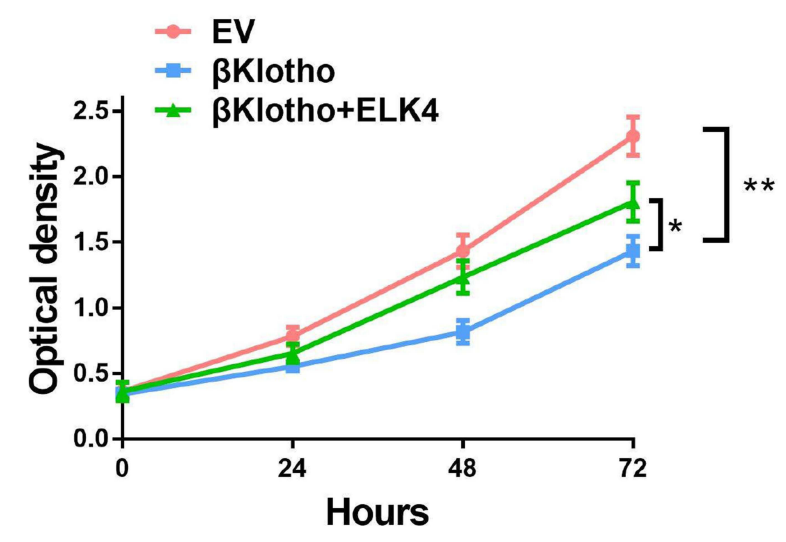

\section{C}

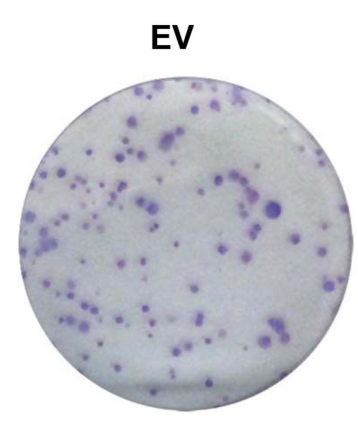

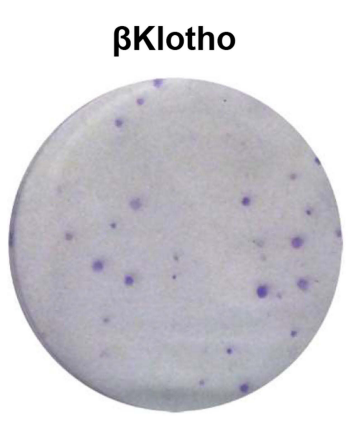
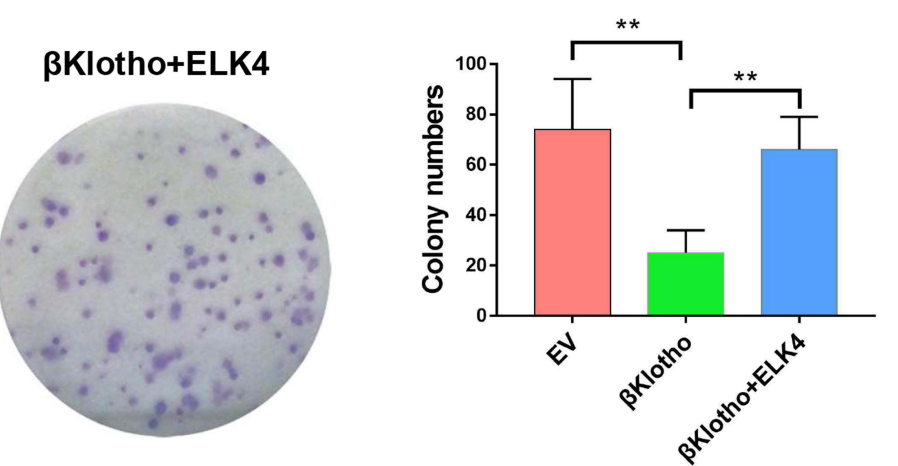

Figure I $\beta$ Klotho overexpression down-regulated the expression of ELK4, and the elevated $\beta K$ lotho expression inhibited the proliferation of $C 4-2 B$ cell line, which can be reversed by the overexpression of ELK4. The C4-2B cell line was transfected with empty vector (GV230-CON083), GV230- $\beta$ Klotho ( $\beta K$ Iotho) or GV230- $\beta$ Klotho plus GV230-ELK4 plasmids ( $\beta$ Klotho+ELK4). (A) The Western blotting assay. (B) The CCK-8 assay. (C) The colony formation assay. ${ }^{*} p<0.05$, ** $p<0.01$.

Abbreviations: ELK4, ETS-like transcription factor 4; PARP, poly ADP-ribose polymerase; EV, empty vector.

\section{Relationships Among $\beta$ Klotho, ELK4 Expression and Clinicopathologic}

\section{Parameters}

The Chi-square tests and Fisher's exact tests were used to explore the links between $\beta$ Klotho, EKL4 expression and clinicopathological variables. The result indicated that high $\beta$ Klotho expression was correlated to lower Gleason score $(p=0.011)$, absent distant metastasis $(p=0.029)$ and lower PSA level $(p=0.008)$. On the contrast, high ELK4 overexpression was correlated to distant metastasis $(p=0.034)$ and higher PSA $(p=0.036)$. No significant differences were indicated for other clinicopathologic parameters (Table 1).

\section{The Prognostic Values of $\beta K$ lotho and ELK4 Expression in PCa Patients}

The overall survival and cancer-specific survival subject to the $\beta$ Klotho expression and ELK4 expression were, respectively, investigated by the Kaplan-Meier method. High $\beta$ Klotho expression predicted favorable prognosis in both overall survival (Figure 4A, $p<0.001$ ) and cancerspecific survival (Figure $4 \mathrm{~B}, p=0.003$ ) in PCa patients, and low ELK4 expression also indicated favorable overall survival (Figure 5A, $p<0.001$ ) and cancer-specific survival (Figure 5B, $p=0.006$ ) in PCa patients.

The cox-regression analysis was applied to determine predictive values of $\beta$ Klotho expression, ELK4 expression and other variables for the prognosis of PCa patients. The univariate analysis result revealed that $\beta$ Klotho expression $(p<$ $0.001)$, ELK4 expression $(p<0.001)$, T stage $(p=0.005)$, Gleason score $(p=0.027)$, PSA $(p=0.002)$ and distant metastasis $(p=0.001)$ were related to the prognosis of $\mathrm{PCa}$ (Figure 4C, Table 2). Further multivariate analysis recognized $\beta$ Klotho expression $\quad(\mathrm{HR}=0.299, \quad 95 \% \quad \mathrm{CI}=126-0.708$, $p=0.006)$, distant metastasis $(\mathrm{HR}=2.326,95 \% \mathrm{CI}=1.032-$ 5.245, $p=0.042)$ and PSA level $(\mathrm{HR}=2.153,95 \% \mathrm{CI}=1.136-$ 
A

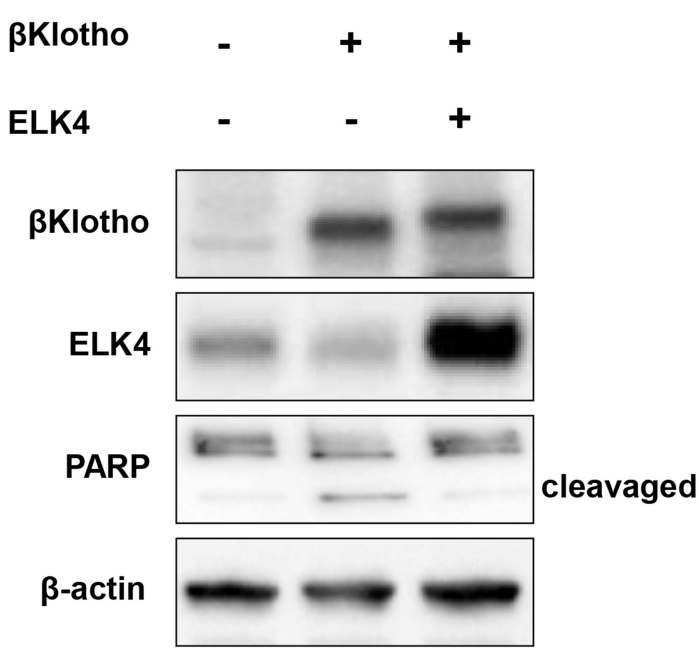

B

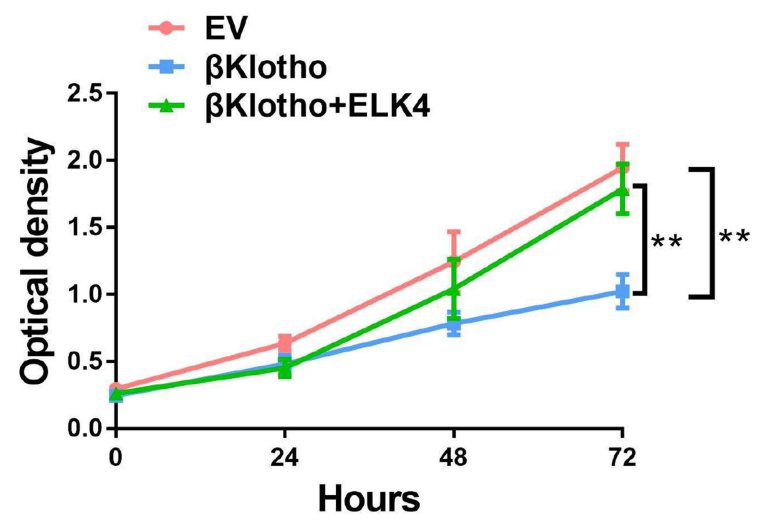

C

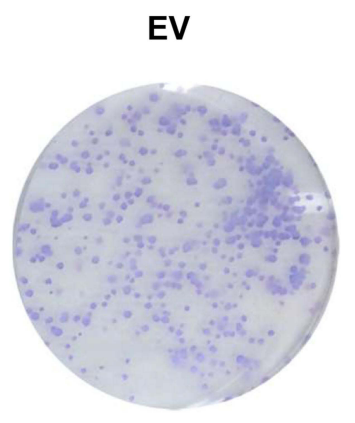

ßKlotho

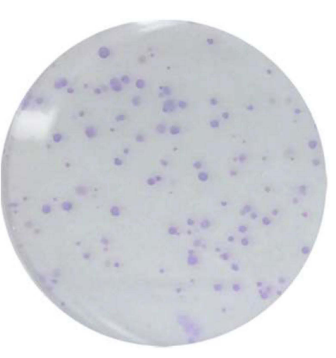

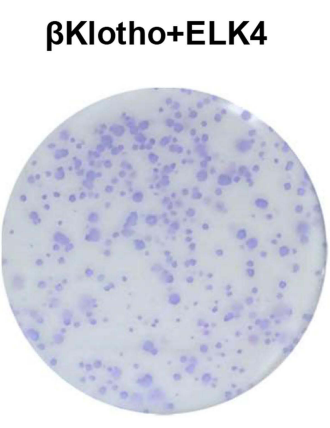

Figure $2 \beta K$ Kotho overexpression down-regulated the expression of ELK4, and the elevated $\beta$ Klotho expression inhibited the proliferation of PC 3 cell line, which can be reversed by the overexpression of ELK4. The PC3 cell line was transfected with empty vector (GV230-CON083), GV230- $\beta$ Klotho ( $\beta K$ lotho) or GV230- $\beta$ Klotho plus GV230-ELK4 plasmids ( $\beta$ Klotho+ELK4). (A) The Western blotting assay. (B) The CCK-8 assay. (C) The colony formation assay. $* * p<0.01$.

Abbreviations: ELK4, ETS-like transcription factor 4; PARP, poly ADP-ribose polymerase; EV, empty vector.

4.080, $p=0.019$ ) as independent prognostic factors in $\mathrm{PCa}$, referring to $\beta$ Klotho expression (Figure $4 \mathrm{D}$, Table 2 ). In addition, the ELK4 expression $(\mathrm{HR}=3.400,95 \% \mathrm{CI}=1.354-8.540$, $p=0.009)$, distant metastasis $(\mathrm{HR}=2.323,95 \% \quad \mathrm{CI}=1.042-$ $5.179, p=0.039)$ and PSA level $(\mathrm{HR}=2.005,95 \% \mathrm{CI}=1.022-$ $3.935, p=0.043$ ) were also considered as independent prognostic factors, referring to ELK4 expression (Figure 5C, Table 2).

We further detected the expressions of $\beta \mathrm{K}$ lotho and ELK4 in the subgroups classified by metastasis status and PSA levels. The results showed that high $\beta$ Klotho expression was related to absent distant metastasis (Figure 4E) and lower PSA level (Figure 4F), whereas high ELK4 expression was related to present distant metastasis (Figure 5D), and higher PSA level (Figure 5E).

\section{Discussion}

$\mathrm{PCa}$, the second most common malignancies in male genitourinary system, has become one of the foremost cancer- related mortality worldwide. ${ }^{23-25}$ Although the elevation of PSA is usually recognized as an indicator of $\mathrm{PCa}$ in clinical diagnosis, its role in predicting the prognosis is limited. PCa can be classified into low-risk type and highrisk type according to their biologic behavior, and different therapeutic schedules are settled for each cancer type and different prognoses are expected. ${ }^{7}$ High-risk PCa calls for radical prostatectomy or radiotherapy, but one of the prominent strategic treatment for low-risk PCa is active surveillance before progression. However, how to precisely predict the prognosis of individual $\mathrm{PCa}$ patients to adjust the therapy strategy remains unclear.

A series of studies have been conducted to exploit promising biomarkers for prognosis prediction of PCa. Hoter et al concluded that members of heat shock proteins (HSPs) were involved in the tumor aggressiveness and prognosis of $\mathrm{PCa},{ }^{26}$ and studies conducted by $\mathrm{Ni}$ et al identified tenascin- $\mathrm{C}$ as a potential cancer-associated 


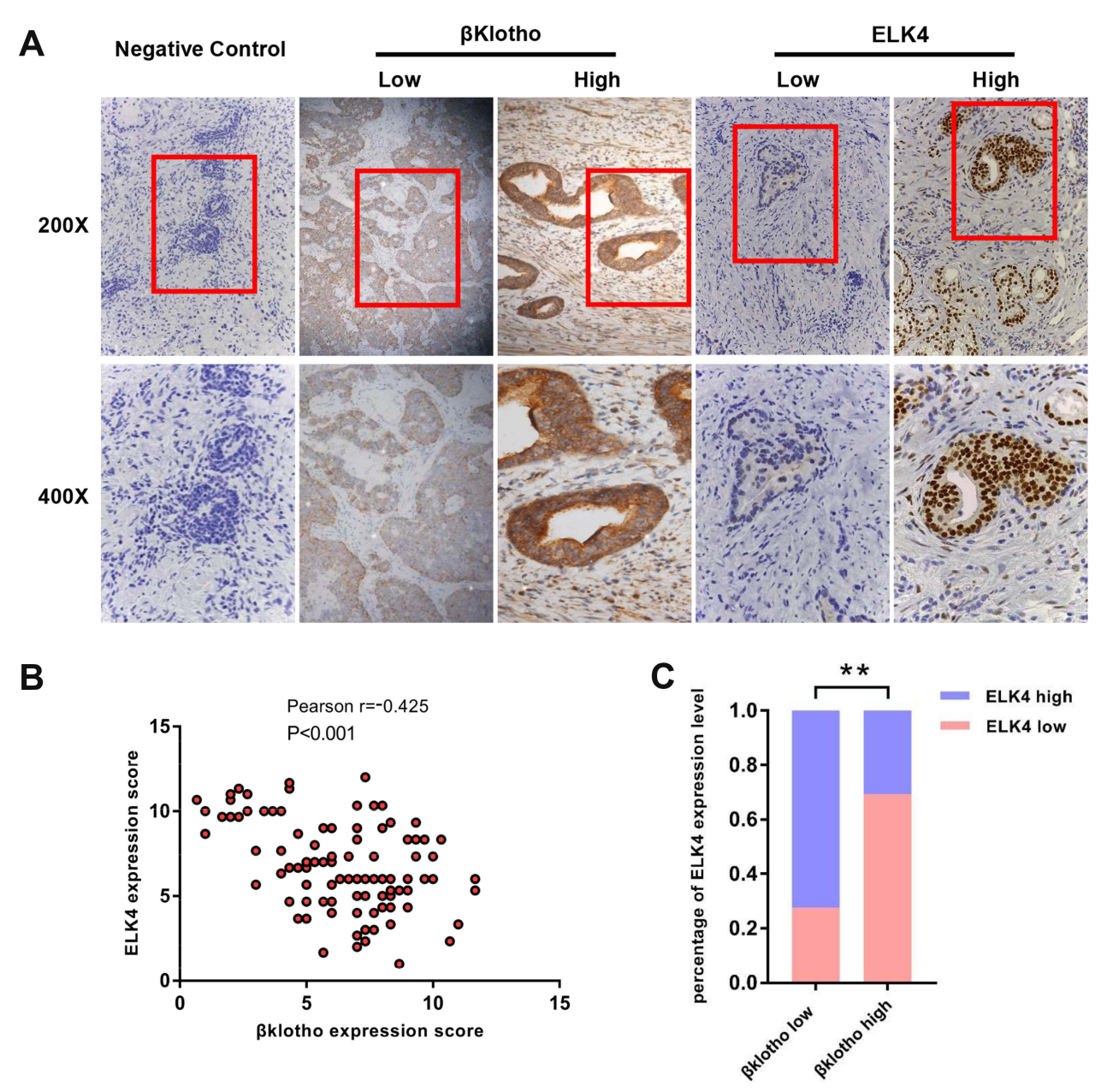

Figure 3 The expression of $\beta$ Klotho and ELK4 in PCa tissues by IHC. (A) Representative immunohistochemical staining images of negative control, $\beta$ Klotho and ELK4 in PCa samples (negative control was stained with primary antibody absence). (B) The expression relationship between $\beta K$ lotho and ELK4 demonstrated by scatter plot. (C) The percentage of ELK4 expression in low and high $\beta$ Klotho expression groups. $*^{*} p<0.01$.

Abbreviations: ELK4, ETS-like transcription factor 4; IHC, immunohistochemistry.

fibroblast marker that predicted poor survival outcome in PCa. ${ }^{27}$ However, none of these candidate biomarkers have been successfully accessed in clinical application.

Several studies have been showed that $\beta$ Klotho was involved in the initiation and progression in multiple cancers. $^{10,28}$ Moreover, reduced $\beta$ Klotho expression in PCa tissues has been proven by our previous study, ${ }^{15}$ indicating the potential tumor-inhibitory role of $\beta$ Klotho in PCa. As the continuation of our previous research, we investigated the prognostic role of $\beta \mathrm{K}$ lotho in $\mathrm{PCa}$. In this study, we identified that $\beta$ Klotho overexpression was associated with favorable clinicopathologic features and predicted favorable prognosis in PCa. Our findings were in accordance with a recent study conducted by Li et al, they found that higher $\beta$ Klotho level was associated with longer overall survival and progression-free survival in non-small cell lung cancer (NSCLC). ${ }^{14}$ However, another study revealed that elevated $\beta \mathrm{K}$ lotho expression was associated with poor progression-free survival of non-muscleinvasive bladder cancer (NMIBC). ${ }^{29}$ This inconsistency may be attributed to multiple aspects of each study (such as sample size, study method, IHC interpretation, etc.) and the context-dependent manner of $\beta$ Klotho in the initiation and progression of human tumors. The association between $\beta$ Klotho expression and the survival benefit for PCa should be further evaluated in larger multiinstitutional trials.

Our previous studies have shown that $\beta$ klotho overexpression could inhibit the proliferation of $\mathrm{PCa}$ cells and endometrial cancer cells through inactivating phosphorylation of ERK signaling. ${ }^{13,15}$ Moreover, as the downstream transcription factor of ERK signaling, ELK4 has been reported to play an oncogenic role in PCa cells, and the expression of ELK4 in PCa was higher than that of benign 
Table I Relationships Between $\beta$ Klotho, EKL4 and Clinicopathological Variables

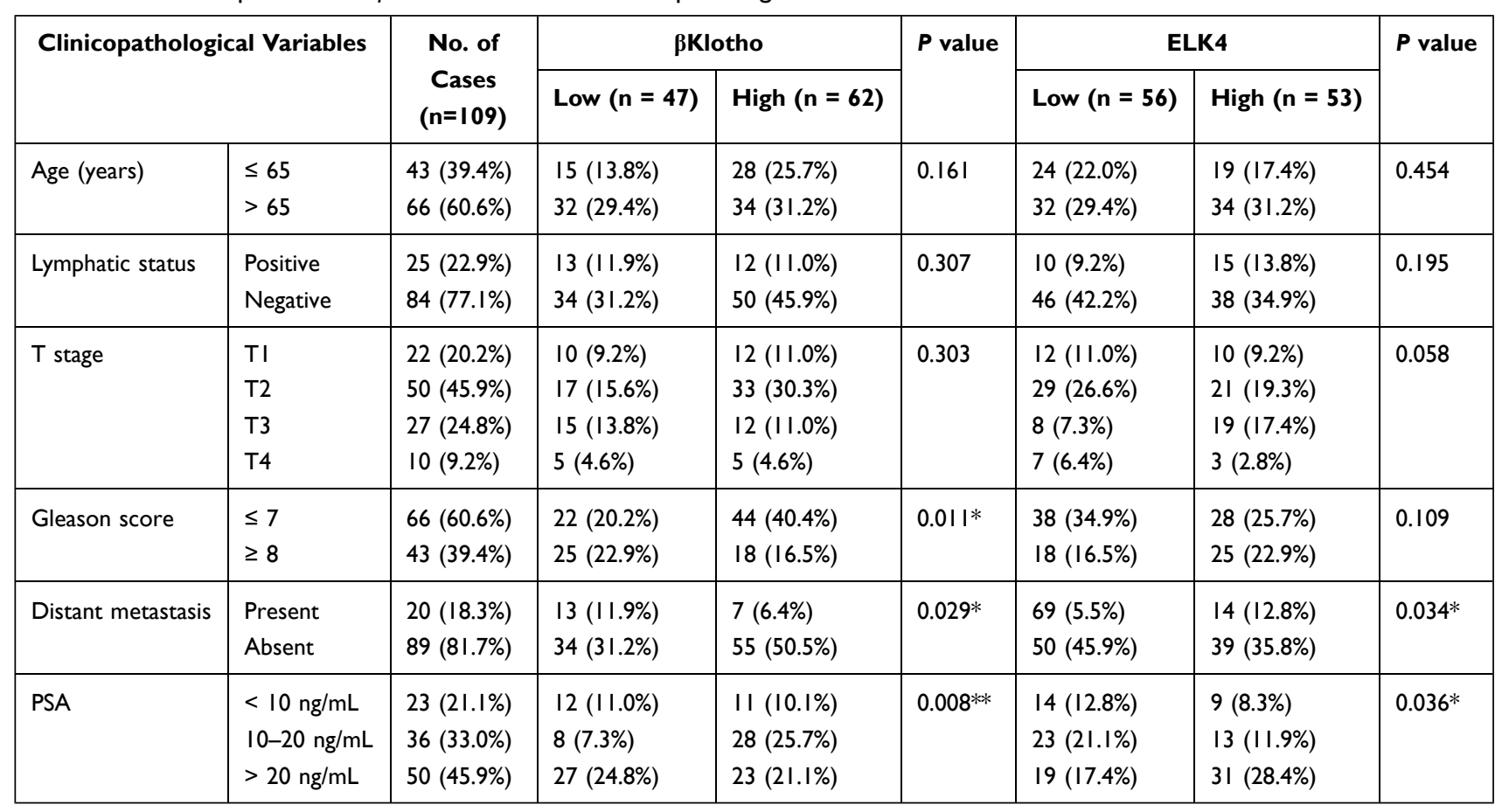

Notes: $*_{p}<0.05, *_{p}<0.01$.

Abbreviations: T, tumor; PSA, prostate-specific antigen; ELK4, ETS-like transcription factor 4.

prostatic hyperplasia. ${ }^{16}$ In addition, the SLC45A3-ELK4 chimeric transcripts were identified in $\mathrm{PCa}$ and interference with the SLC45A3-ELK4 expression significantly inhibited the proliferation of PCa cells. ${ }^{30}$ Based on the above evidence, we are curious as to whether there is a regulatory relationship between $\beta$ Klotho and ELK4. In
A

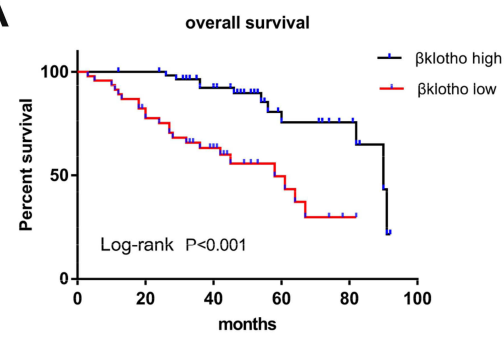

D

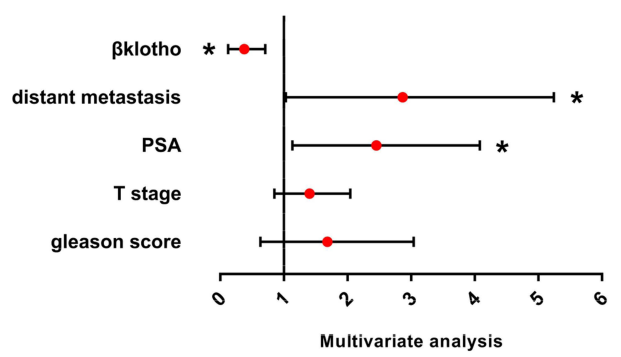

B

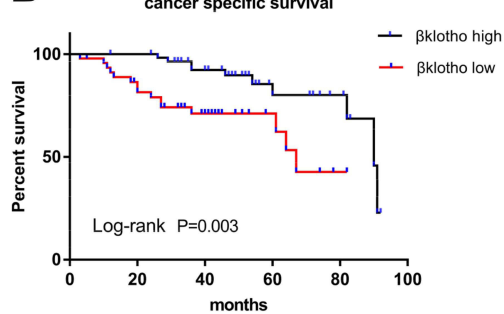

E

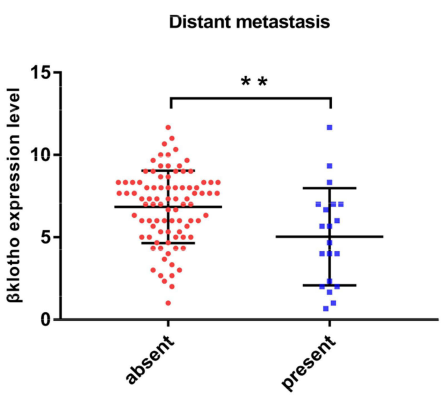

C

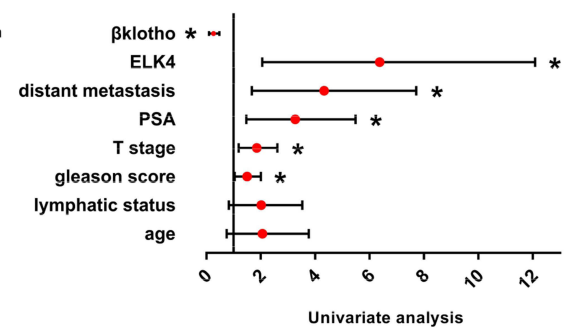

$\mathbf{F}$

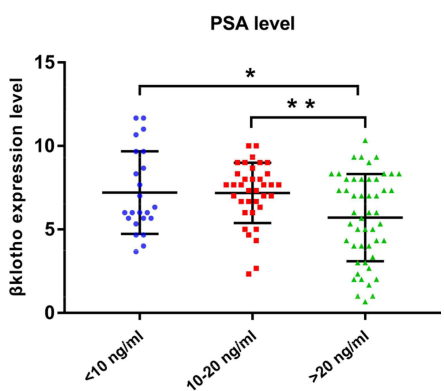

Figure 4 The prognosis prediction role of $\beta K$ lotho in PCa. (A) The Kaplan-Meier survival curve revealed the relationship between $\beta K$ lotho and overall survival of PCa. (B) The Kaplan-Meier survival curve revealed the relationship between $\beta$ Klotho and cancer specific survival of PCa. (C) The univariate analysis demonstrated the predictable role of different clinicopathological variables in the prognosis of PCa. (D) The multivariate analysis demonstrated the independent predictable role of $\beta K$ lotho and different clinicopathological variables in the prognosis of PCa. (E) The different $\beta K$ lotho expression level in subgroups classified by distant metastasis. (F) The different $\beta$ Klotho expression level in subgroups classified by PSA level. ${ }^{*} p<0.05$, $* * p<0.01$.

Abbreviations: PCa, prostate cancer; T, tumor; PSA, prostate-specific antigen; ELK4, ETS-like transcription factor 4. 

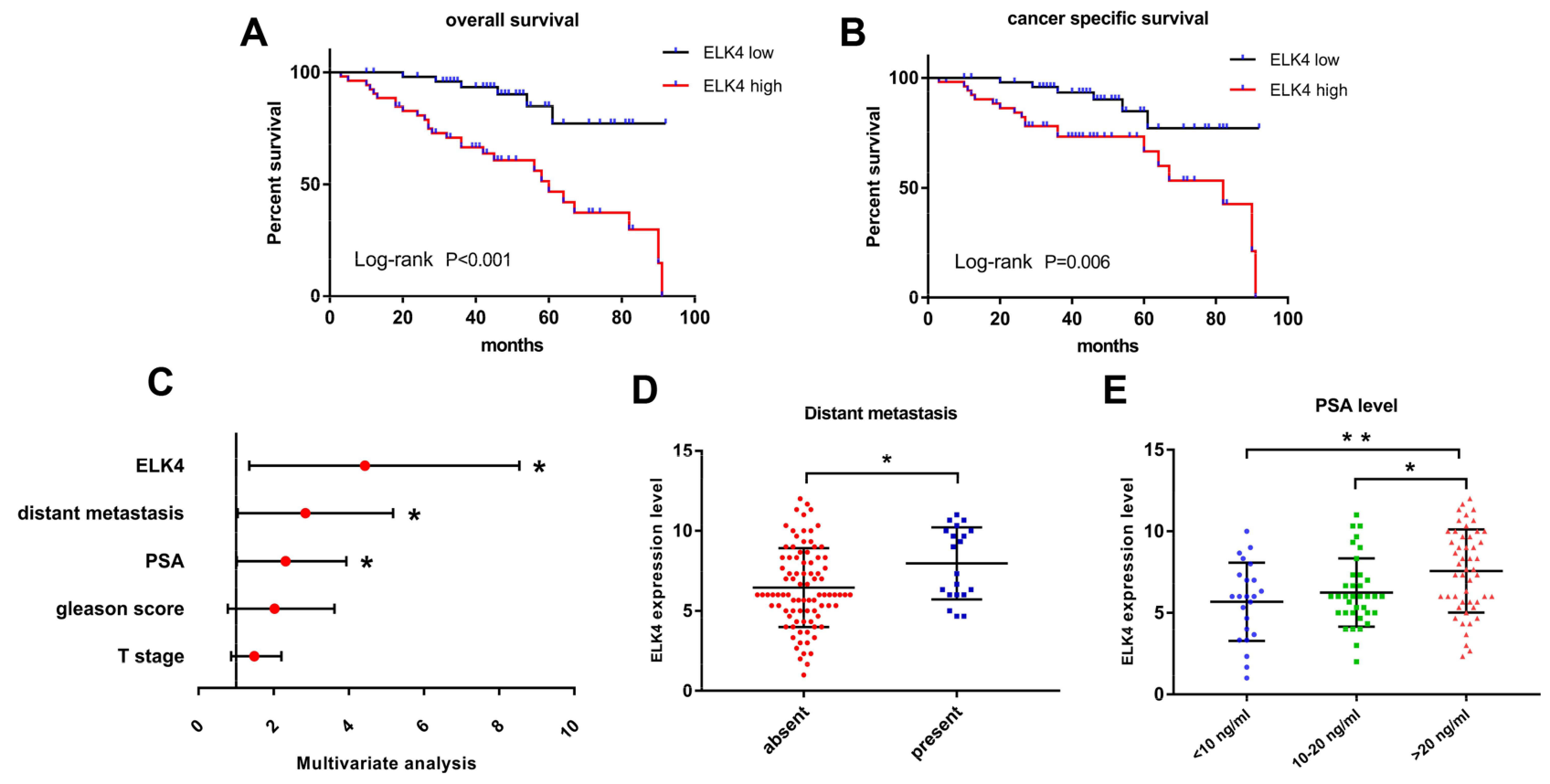

Figure 5 The prognosis prediction role of ELK4 in PCa. (A) The Kaplan-Meier survival curve revealed the relationship between ELK4 and overall survival of PCa. (B) The Kaplan-Meier survival curve revealed the relationship between ELK4 and cancer specific survival of PCa. (C) The multivariate analysis demonstrated the independent predictable role of ELK4 and different clinicopathological variables in the prognosis of PCa. (D) The different $\beta$ Klotho expression level in subgroups classified by distant metastasis. (E) The different $\beta$ Klotho expression level in subgroups classified by PSA level. $*_{p}<0.05, *^{*} p<0.01$.

Abbreviations: PCa, prostate cancer; T, tumor; PSA, prostate-specific antigen; ELK4, ETS-like transcription factor 4.

this study, we revealed the negative correlation between $\beta$ Klotho expression and ELK4 expression in PCa tissues. And in contrast to $\beta$ Klotho, ELK4 expression predicted poor prognosis in PCa patients. Further in vitro experiments demonstrated that $\beta$ Klotho overexpression inhibited cell proliferation by downregulating ELK4. However, how a transmembrane protein regulates the expression of a transcriptional factor and what is the specific mechanism link the $\beta$ Klotho and ELK4 remain elusive. By retrieving literatures, an article titled "BAI1 Suppresses Medulloblastoma Formation by Protecting p53 from Mdm2-Mediated Degradation"31 has provided us with

Table 2 Univariate and Multivariate Analysis of Prognosis for Patients with PCa

\begin{tabular}{|c|c|c|c|c|c|c|c|c|c|}
\hline & \multicolumn{2}{|c|}{ Univariate Analysis } & \multirow[t]{2}{*}{$P$ value } & \multicolumn{2}{|c|}{$\begin{array}{l}\text { Multivariate Analysis } \\
\text { ( } \beta \text { Klotho) }\end{array}$} & \multirow[t]{2}{*}{$P$ value } & \multicolumn{2}{|c|}{$\begin{array}{l}\text { Multivariate Analysis } \\
\text { (ELK4) }\end{array}$} & \multirow[t]{2}{*}{$P$ value } \\
\hline & HR & $95 \% \mathrm{Cl}$ & & HR & $95 \% \mathrm{Cl}$ & & HR & $95 \% \mathrm{Cl}$ & \\
\hline Age & 1.675 & $0.745-3.768$ & 0.212 & & & & & & \\
\hline Lymphatic status & 1.710 & $0.829-3.525$ & 0.157 & & & & & & \\
\hline T stage & 1.762 & $1.188-2.613$ & $0.005^{* *}$ & 1.319 & $0.85 I-2.046$ & 0.216 & 1.385 & $0.869-2.208$ & 0.171 \\
\hline Gleason score & 1.445 & $1.043-2.002$ & $0.027^{*}$ & 1.300 & $0.624-3.007$ & 0.433 & 1.679 & $0.778-3.622$ & 0.186 \\
\hline PSA & 2.838 & I.468-5.488 & $0.002 * *$ & 2.153 & $1.136-4.080$ & $0.019 *$ & 2.005 & $1.022-3.935$ & $0.043 *$ \\
\hline Distant metastasis & 3.595 & I.675-7.7I7 & $0.00 I^{* *}$ & 2.326 & $1.032-5.245$ & $0.042 *$ & 2.323 & $1.042-5.179$ & $0.039 *$ \\
\hline$\beta$ klotho & 0.219 & $0.100-0.479$ & $<0.001 * * *$ & 0.299 & $0.126-0.708$ & $0.006 * *$ & & & \\
\hline ELK4 & 4.980 & $2.052-12.088$ & $<0.001 * * *$ & & & & 3.400 & $1.354-8.540$ & $0.009 * *$ \\
\hline
\end{tabular}

Notes: $*_{p}<0.05, *_{p}<0.01,{ }^{*} * p<0.001$.

Abbreviations: PCa, prostate cancer; T, tumor; PSA, prostate-specific antigen; ELK4, ETS-like transcription factor 4. 
some enlightenment. They revealed an interaction between transmembrane protein BAI1 and transcriptional factor MDM2 by using Co-Immunoprecipitation. Their data made us assume that there may exist similar regulatory pattern between $\beta$ Klotho and ELK4. However, the research regarding the mechanism is complex and may involve other factors or pathways, and we will endeavor to address this issue in the future studies.

\section{Conclusion}

In conclusion, for the first time, this study demonstrated that $\beta$ Klotho inhibits proliferation of PCa cells by downregulating ELK4 and predicts favorable prognosis in $\mathrm{PCa}$ patients. The $\beta$ Klotho and ELK4 may serve as potential targets for therapeutic purpose and prognosis prediction in the future.

\section{Acknowledgments}

This research was supported by grants from the Natural Science Foundation of Shandong Province (no. ZR2020QH068), Shandong Province Key Research and Development Projects (no. 2016GSF201147 and no. 2016GSF201156), and the Science and Technology Development Program of Jinan (no. 201704127).

\section{Disclosure}

The authors declare that there is no conflict of interest regarding the publication of this paper.

\section{References}

1. Siegel RL, Miller KD, Jemal A. Cancer statistics, 2020. CA Cancer J Clin. 2020;70(1):7-30.

2. Carlsson SV, Vickers AJ. Screening for prostate cancer. Med Clin North Am. 2020;104(6):1051-1062. doi:10.1016/j.mena.2020.08.007

3. Ye DW, Zhu Y. Prostate cancer and prostatic diseases best of China, 2018. Prostate Cancer Prostatic Dis. 2019;22(1):1-2. doi:10.1038/ s41391-018-0117-y

4. Blackwelder R, Chessman A. Prostate cancer screening: shared decision-making for screening and treatment. Prim Care. 2019;46 (1):149-155. doi:10.1016/j.pop.2018.10.012

5. Yu A, Guo K, Qin Q, Xing C, Zu X. Clinicopathological and prognostic significance of osteopontin expression in patients with prostate cancer: a systematic review and meta-analysis. Biosci Rep. 2021. doi:10.1042/BSR20203531

6. Li T, Wang Q, Hong X, et al. RRBP1 is highly expressed in prostate cancer and correlates with prognosis. Cancer Manag Res. 2019;11:3021-3027. doi:10.2147/CMAR.S186632

7. Jiang F, Dai L, Yang S, et al. Increasing of FKBP9 can predict poor prognosis in patients with prostate cancer. Pathol Res Pract. 2020;216 (1):152732. doi:10.1016/j.prp.2019.152732

8. Xie Y, Su N, Yang J, et al. FGF/FGFR signaling in health and disease. Signal Transduct Target Ther. 2020;5(1):181.

9. Kuro-o M. Klotho and BKlotho. Adv Exp Med Biol. 2012;728:25-40.
10. Kuro OM. The Klotho proteins in health and disease. Nat Rev Nephrol. 2019;15(1):27-44. doi:10.1038/s41581-018-0078-3

11. Kale A, Sankrityayan H, Anders HJ, Gaikwad AB. Epigenetic and non-epigenetic regulation of Klotho in kidney disease. Life Sci. 2021;264:118644. doi:10.1016/j.1fs.2020.118644

12. Lin ZZ, Hsu C, Jeng YM, et al. Klotho-beta and fibroblast growth factor 19 expression correlates with early recurrence of resectable hepatocellular carcinoma. Liver Int. 2019;39(9):1682-1691. doi:10.1111/liv.14055

13. Liu Z, Qi S, Zhao X, et al. Metformin inhibits 17beta-estradiolinduced epithelial-to-mesenchymal transition via $\beta$ Klotho-related ERK1/2 signaling and AMPKalpha signaling in endometrial adenocarcinoma cells. Oncotarget. 2016;7(16):21315-21331. doi:10.18632/oncotarget.7040

14. Li F, Li X, Li Z, Ji W, Lu S, Xia W. $\beta$ Klotho is identified as a target for theranostics in non-small cell lung cancer. Theranostics. 2019;9 (25):7474-7489. doi:10.7150/thno.35582

15. Liu Z, Zhang H, Ding S, et al. $\beta$ Klotho inhibits androgen/androgen receptor-associated epithelial-mesenchymal transition in prostate cancer through inactivation of ERK1/2 signaling. Oncol Rep. 2018;40 (1):217-225.

16. Makkonen $H$, Jääskeläinen $T$, Pitkänen-Arsiola $T$, et al. Identification of ETS-like transcription factor 4 as a novel androgen receptor target in prostate cancer cells. Oncogene. 2008;27(36):4865-4876. doi:10.1038/onc.2008.125

17. Maurice D, Costello P, Sargent M, Treisman R. ERK signaling controls innate-like $\mathrm{CD} 8(+) \mathrm{T}$ cell differentiation via the ELK4 (SAP-1) and ELK1 transcription factors. J Immunol. 2018;201 (6):1681-1691. doi:10.4049/jimmunol.1800704

18. Xie L. MKL1/2 and ELK4 co-regulate distinct serum response factor (SRF) transcription programs in macrophages. BMC Genomics. 2014;15:301. doi:10.1186/1471-2164-15-301

19. Liu Q, Zhu L, Liu X, et al. TRA2A-induced upregulation of LINC00662 regulates blood-brain barrier permeability by affecting ELK4 mRNA stability in Alzheimer's microenvironment. RNA Biol. 2020;17(9):1293-1308. doi:10.1080/ 15476286.2020.1756055

20. Long R, Liu Z, Li J, Zhang Y, Yu H. HCG11 up-regulation induced by ELK4 suppressed proliferation in vestibular schwannoma by targeting miR-620/ELK4. Cancer Cell Int. 2021;21(1):5. doi:10.1186/ s12935-020-01691-0

21. Zhu Z, Song J, Guo Y, et al. LAMB3 promotes tumour progression through the AKT-FOXO3/4 axis and is transcriptionally regulated by the BRD2/acetylated ELK4 complex in colorectal cancer. Oncogene. 2020;39(24):4666-4680. doi:10.1038/s41388-0201321-5

22. Shaikhibrahim Z, Lindstrot A, Langer B, Buettner R, Wernert N. Differential expression of ETS family members in prostate cancer tissues and androgen-sensitive and insensitive prostate cancer cell lines. Int J Mol Med. 2011;28(1):89-93.

23. Ha Chung B, Horie S, Chiong E. The incidence, mortality, and risk factors of prostate cancer in Asian men. Prostate Int. 2019;7(1):1-8. doi:10.1016/j.prnil.2018.11.001

24. Terada N, Akamatsu S, Kobayashi T, Inoue $\mathrm{T}$, Ogawa $\mathrm{O}$, Antonarakis ES. Prognostic and predictive biomarkers in prostate cancer: latest evidence and clinical implications. Ther Adv Med Oncol. 2017;9(8):565-573. doi:10.1177/1758834017719215

25. Filella X, Foj L. Novel biomarkers for prostate cancer detection and prognosis. Adv Exp Med Biol. 2018;1095:15-39.

26. Hoter A, Rizk S, Naim HY. The multiple roles and therapeutic potential of molecular chaperones in prostate cancer. Cancers. 2019;11(8):1194. doi:10.3390/cancers11081194

27. Ni WD, Yang ZT, Cui CA, Cui Y, Fang LY, Xuan YH. Tenascin-C is a potential cancer-associated fibroblasts marker and predicts poor prognosis in prostate cancer. Biochem Biophys Res Commun. 2017;486(3):607-612. doi:10.1016/j.bbrc.2017.03.021 
28. Somm E, Henry H, Bruce SJ, et al. $\beta$ Klotho deficiency shifts the gutliver bile acid axis and induces hepatic alterations in mice. $\mathrm{Am}$ J Physiol Endocrinol Metab. 2018;315(5):E833-E847. doi:10.1152/ ajpendo.00182.2018

29. Hori S, Miyake M, Onishi S, et al. Clinical significance of alpha and $\beta$ Klotho in urothelial carcinoma of the bladder. Oncol Rep. 2016;36 (4):2117-2125. doi:10.3892/or.2016.5053
30. Qin F, Zhang Y, Liu J, Li H. SLC45A3-ELK4 functions as a long non-coding chimeric RNA. Cancer Lett. 2017;404:53-61. doi:10.1016/j.canlet.2017.07.007

31. Zhu D, Osuka S, Zhang Z, et al. BAI1 suppresses medulloblastoma formation by protecting p53 from Mdm2-mediated degradation. Cancer Cell. 2018;33(6):1004-1016.e1005. doi:10.1016/j. ccell.2018.05.006

\section{Publish your work in this journal}

Cancer Management and Research is an international, peer-reviewed open access journal focusing on cancer research and the optimal use of preventative and integrated treatment interventions to achieve improved outcomes, enhanced survival and quality of life for the cancer patient.
The manuscript management system is completely online and includes a very quick and fair peer-review system, which is all easy to use. Visit http://www.dovepress.com/testimonials.php to read real quotes from published authors. 